\title{
How we wrote a landmark nasopharyngeal intergroup phase III protocol in North America
}

\author{
Muhyi Al-Sarraf
}

Received: 11 April 2012 / Accepted: 12 April 2012 /Published online: 2 May 2012

(C) Springer-Verlag 2012

\section{Introduction and background}

The results of the North America Intergroup 0099 trial [1] represented a key turning point and a milestone for the management of patients with locally advanced nasopharyngeal cancer (NPC) and heralded a new era of the combined modality treatment for these patients. The sequence of concurrent chemoradiotherapy $(\mathrm{CT}+\mathrm{RT})$ with cisplatin $(\mathrm{P})$ given with radiotherapy (RT) every 3 weeks followed by adjuvant chemotherapy (CT) with cisplatin and 5FU (PF) for an additional three cycles produced complete clearance of about $90 \%$ of tumors and a 5 -year survival rate of about $75 \%$ of patients who had locally advanced NPC (stage III, $10 \%$; stage, IV $90 \%$ ). At the same time, it raised the question about the most effective and the least toxic sequence of concurrent $\mathrm{CT}+\mathrm{RT}$ and effective combination $\mathrm{CT}$.

At the time the trial was designed, we knew that NPC is a unique malignant head and neck cancer with clinical, demographic, and geographic features distinct from other head and neck epithelial malignancies. NPC occurs in patients who often are younger than patients with other head and neck cancers and in most cases is not related to tobacco use. Nasopharyngeal cancer has a worldwide distribution. Its incidence, however, varies with geographical location. It is rare in North American and European Caucasians. In the USA, the incidence is about 1 per 100,000 . It is 0.8 per

\footnotetext{
M. Al-Sarraf $(\bowtie)$

William Beaumont Hospital,

Royal Oak, MI, USA

e-mail: mealsarraf@aol.com

M. Al-Sarraf

Department of Medicine,

2810 Birchwood CT,

Bloomfield Hills, MI 48302-0900, USA
}

100,000 in males and 0.3 per 100,000 in females, with a male-to-female ratio of $2.4: 1$. The peak age is between 50 and 59 years. There are also geographic regions where NPC is endemic, such as in Southeast Asia, Northern Africa, and parts of the Mediterranean Basin [2]. In China and Southeast Asia, the incidence is about $25-30$ per 100,000 . It is also high in the Eskimos (the incidence is intermediate in the Middle East and North Africa). In endemic areas, the histopathology is almost exclusively poorly or undifferentiated carcinoma (WHO II, A and B), while in North America up to $25 \%$ of patients present with squamous cell carcinoma (WHO I); the rest are WHO II.

In light of the uncommon incidence of NPC in North America, it was obvious that any phase III trial would need to have intergroup cooperation with full participation of the majority of the institutions in the cooperative groups. We also counted on the greater NPC incidence in some parts of California (the Chinese community in San Francisco and the Asian population of Los Angeles and Southern California), New York City, and the Texas Gulf regions that became home to Southeast Asians after the end of the Vietnam conflict in 1975.

\section{Historical points: results of phase II trials}

The majority of the patients who had NPC in North America presented with stage III or IV disease. The incidence of systemic metastasis at presentation or after failure of the local treatment(s) was higher than observed in other head and neck $(\mathrm{H} \& \mathrm{~N})$ cancer sites. We knew that NPC is highly sensitive to radiotherapy and had begun to think that it was also sensitive to chemotherapy [3-9]. RT had been the mainstay of the management of locally advanced nasopharyngeal cancers (stages III and IV), and while the results of 
RT in patients who had stage IV disease was somewhat better than seen in other H\&N cancers, the results were still disappointing. Even in the face of relatively high local control rates following RT, the overall 5 -year survival of stage IV was $<40 \%$ across the world, and the logic of trying to add CT became compelling.

With the discovery of cisplatin as an active agent in patients with $\mathrm{H} \& \mathrm{~N}$ cancers (including NPC) in the mid 1970s and the "bonus" that it acted as a radiosensitizer without pronounced intensification of mucositis, the combination of cisplatin (or cisplatin-containing regimens) with RT formed the backbone of several clinical trials. Combination CT was given as induction before RT or as adjuvant after RT. Single-agent cisplatin was administered concurrently with RT in phase II studies. The results of these phase II trials in patients with stage IV NPC improved the 5-year survival to about $50-55 \%$ of these patients [10-12].

\section{Choosing the right, acceptable, and wining experimental arm}

As in other intergroup trials, one group was selected by NCI as the lead group; for this trial, it was the Southwest Oncology Group (SWOG). I was chosen the principal investigator and was tasked to write the first draft of the protocol. Because of the limited incidence of NPC in North America, I suspected this might be the only phase III trial for advanced nasopharyngeal cancer to be conducted in my lifetime. I also knew that with stage IV disease, the addition of limited intensity CT before, during, or post-RT likely would only result in a modest improvement in survival (10-15\%) as compared to RT alone, and even if we were lucky, a trial based on such treatment likely would need a much larger number of evaluable patients and longer time of accrual for the trial to show a significant difference. In addition, the trial had to be acceptable by the majority of the participating institutions and by the majority of investigators, especially the radiation oncologists. I thought the ideal experimental arm would have been induction CT followed by concurrent cisplatin and RT, which I was testing (with a complete clearance rate in locally advanced NPC patients of $100 \%$ and a projected 5-year survival of about $90 \%$ on an experimental institutional protocol) for patients with locally advanced H\&N cancers including NPC. However, some physicians were reluctant to treat tumors that had been "down-staged" with the then relatively new concept of concurrent therapy (especially those tumors that had a complete clinical response). Also, induction CT precluded the head-to-head comparison of response rates and possible side effects. After considerable discussion, we decided that the experimental arm would consist of concurrent cisplatin $100 \mathrm{mg} / \mathrm{m}^{2}$ on days 1,22 , and 43 of RT followed by the combination of cisplatin $80 \mathrm{mg} / \mathrm{m}^{2}$ and $5 \mathrm{FU} 4,000 \mathrm{mg} / \mathrm{m}^{2}$ as a 96-h continuous infusion after the end of RT and every 28 days thereafter, for a total of three additional courses. The doses of both cisplatin and 5FU were reduced by $20 \%$, and the repeat of these courses was prolonged to every 28 days (instead of every 21 days) to reduce the risk of possible toxicities in this sequence of combined modality treatment.

\section{Issues to overcome and agree upon}

\section{Pretreatment workup}

Accurate staging was particularly essential for this protocol because of the complex anatomy of the nasopharyngeal region and the greater risk of systemic involvement than is seen in association with other sites of head and neck cancer. In addition to a detailed history and physical examination (including performance status) and blood tests (including creatinine clearance), patients had to have a chest X-ray and, if their serum enzymes were elevated, liver or bone scan. Three-dimensional views of the nasopharynx, base of skull, and the neck by CAT scan or MRI were mandatory. A bilateral audiogram, nasopharyngoscopy with satisfactory biopsy of the primary lesion, plus dental clearance before the start of radiotherapy were all required. Initially, triple endoscopy (pharynx, lung, and esophagus) was required, but on 1 December 1994, the study was amended to delete the triple endoscopy requirement. All patients were required to provide written informed consent before registration on the study. Care was randomized and stratified according to $\mathrm{T}$ and $\mathrm{N}$ categories (not the grouped stage), WHO histopathological classification, and performance status.

\section{Engineering the overall approval}

In previous intergroup trials, it was customary for the selected "lead" group, in this case the SWOG, to have the principal investigator(s) write the first draft of the protocol, submit it to the anatomic site committee for approval $(\mathrm{H} \& \mathrm{~N})$, then the full group approval (SWOG), and then submit it to the NCI for the final approval. After NCI approval, the protocol would be sent to other cooperative groups for discussion and potential activation. For this Intergroup phase III randomized trial, it was decided after the anatomic site (the SWOG Head \& Neck Committee) approval to submit to the other cooperative groups for their comments and approval before sending the protocol to the NCI for final approval. I am not sure this was the wisest decision, but it resulted in receiving all kinds of comments and changes from the other investigators. We were able to include the majority of these changes as long as they did not change the intention of this important trial and its principles. 
However, it is important to understand that the protocol that was activated represented a consensus that had its benefits and limits. For example, a major obstacle point of discussion involved the maximum allowed dose to the spinal cord. Some radiation oncologists would only accept a dose of 4,500 cGy, while others were willing to accept 5,000 cGy. The final decision was to limit the spinal cord dose to 4,500 cGy at midline, and this may have limited the amount of radiation delivered to the tumor and thereby influenced the results.

In addition, because of the decision to submit the draft protocol to the other cooperative groups for comment before submission to the NCI, the large number of intense discussions resulted consumed about 2 years between the initial draft of the study to the final approval and activation. Even so, at the last minute, two large institutions withdraw from participation. Each had promised to contribute about 30 patients per year to the study; ironically, one institution did not want patients to receive experimental chemoradiotherapy, while the other did not want patients to receive RT alone (they called it the inferior arm). For a trial based on such an uncommon tumor type, we knew this would inevitably prolong accrual.

\section{Analysis rules}

The accrual goal of the study was 270 patients. Interim analyses were planned after 56 and $78 \%$ of the patients were accrued. To preserve an overall significance level of 0.05 , one-sided tests at 0.003 and 0.004 were planned for the first and second interim analyses. In addition, reporting of the results shortly after closure of the study was to be considered if the results were significant at the 0.005 level. Otherwise, the results were intended to be reported after approximately one more year of follow-up using a significance level of 0.046 [1].

Evaluating and reporting the results

At the time of the first planned interim analysis in October 1995 , the null hypothesis test of no difference in survival for radiotherapy versus chemoradiotherapy was rejected at the 0.001 level. The hazard ratio between radiotherapy alone and combined therapy was 3.28 , which corresponded to the median survival duration of 30 months in the radiotherapyalone arm versus median survival "not reached" in the chemoradiotherapy arm. The SWOG Data and Safety Monitoring Committee reviewed the interim analysis, which included survival, progression, toxicity, and other outcome information, and recommended that the study be closed and reported early [1]. Between May 1989 and early closure of the study on 1 December 1995, 193 patients were registered onto the study: 103 from the Radiation Therapy Oncology
Group, 60 from SWOG, and 30 from the Eastern Cooperative Oncology Group. There was good balance in the prognostic factors, including performance status, tumor and nodal categories, and histology between the two arms. At the time of early closure and analysis for presentation and publication, the median progression-free survival (PFS) for the radiotherapy alone group was 15 months and had not been reached for the combined group. The 3-year actuarial PFS rates were 24 and $69 \%$, respectively $(P<0.001)$. Fortyfive patients treated by radiotherapy alone and 20 treated by chemoradiotherapy had experienced tumor progression. The estimated hazard ratio was 4.34 , with a $95 \%$ confidence interval of 2.47-7.69. The median overall survival durations for the radiotherapy and chemoradiotherapy groups were 34 months and "not reached," respectively. The 3-year survival rates were 47 and $78 \%$, respectively $(P=0.005)$. These were the most significant differences ever obtained and reported in patients with solid tumors!

Preparing for presentation and publications

Because of their importance, we decided to submit these results for presentation at both of the annual meetings of the American Society for Clinical Oncology [13] and the American Society of Therapeutic Oncology [14]. This was done, and both presentations were accepted for the respective plenary sessions of the meetings in 1996 and well received. However, for initial formal publication, the statistician of the protocol and I decided to present the results only for those patients who were known to be "protocol eligible" and not to include an "intent-to-treat" analysis, thereby excluding patients who, in my opinion, had a very minor infraction, such as a delay in obtaining a pre- or post-randomization test like a chest X-ray or blood test. These created differences in the number of patients reported in each of the treatment arms, but did not alter the "take-home message" or the statistical power of the differences between the two treated groups. But when we submitted the manuscript to a wellknown prestigious journal, one of the reviewers raised a concern about the number discrepancy and the manuscript was rejected. Even when we subsequently provided both results (per protocol and intent-to-treat), the manuscript was not accepted. We then submitted a revised manuscript to the Journal of Clinical Oncology where it was rapidly accepted and published.

\section{End results and present practice}

A decade and a half has passed since our initial report and we remain gratified that the clearly superior results of chemoradiotherapy, as was tested in this important study, have largely formed the backbone of care for patients who have 
locoregionally advanced nasopharyngeal carcinoma. We also recognize that unproven modifications are being practiced around the world. Yet, to practice medicine by level 1 criteria based on this trial, cisplatin should be given concurrently during RT, the dose and the number of cycles should not be reduced (unless related to drug-induced side effects), even with complete clearance of locally advanced disease the adjuvant phase of CT should be administered, and the number of cycles of adjuvant $\mathrm{CT}$ should not be reduced from 3 . But that does not mean that all of these concepts are correct. Newer thinking and newer technology should prompt us to ask:

1. Is chemoradiotherapy appropriate for the earlier stage tumors that were not included in this trial?

2. Does the more precise delineation of tumors and normal tissues provided by modern scanners and the ability to deliver more intense (IMRT) and more accurate (IGRT) radiotherapy negate at least some of the value of concurrent chemoradiotherapy?

3. Would changing the sequence of combined treatment to induction $\mathrm{CT}$ followed by the concurrent $\mathrm{CT}+\mathrm{RT}$ alter the results?

4. Would newer agents and regimens be more effective? For example, would a three-agent combination (TPF) for three courses be superior to the two-agent combination (PF) used in the adjuvant phase of INT 0099?

5. Is there a way to better triage patient care? Do all patients need the adjuvant phase of therapy or can we decrease the toxicity of treatment for some?

The balance of proven and potential remains alive in nasopharyngeal cancer.

Conflict of interest The author acknowledges no financial disclosures or conflicts of interest.

\section{References}

1. Al-Sarraf M, LeBlanc M, Giri PG, Fu KK et al (1998) Chemoradiotherapy versus radiotherapy in patients with advanced nasopharyngeal cancer: phase III randomized Intergroup Study 0099. J Clin Oncol 16:1310-1317
2. Yu MC, Yuan JM (2002) Epidemiology of nasopharyngeal carcinoma. Semin Cancer Biol 12:421-429

3. Decker D, Drelichman A, Al-Sarraf M (1983) Chemotherapy for nasopharyngeal carcinoma: a ten-year experience. Cancer 52:602-605

4. Al-Kourainy K, Crissman J, Ensley JF et al (1988) Excellent response to cis-platinum based chemotherapy in patients with recurrent or previously untreated advanced nasopharyngeal carcinoma. Amer J Clin Oncol (CCT) 11:427-430

5. Al-Sarraf M, Pajak TF, Cooper JS et al (1990) Chemoradiotherapy in patients with locally advanced nasopharyngeal carcinoma: a Radiation Therapy Oncology Group study. J Clin Oncol 8(8):1342-1351

6. Giri PG, Al-Sarraf M (2003) Nasopharyngeal cancer. In: Ensley JF, Gutkind S, Jacobs JA, Lippman S (eds) Head and neck emerging perspectives. Academic, San Diego, pp 491-501

7. Benasso M, Sanguineti G, D’Amico M et al (2000) Induction chemotherapy followed by alternating chemo-radiotherapy in stage IV undifferentiated nasopharyngeal carcinoma. $\mathrm{Br} \mathrm{J}$ Cancer 83:1437-1442

8. Rischin D, Corry J, Smith J et al (2002) Excellent disease control and survival in patients with advanced nasopharyngeal cancer treated with chemoradiation. J Clin Oncol 20:1845-1852

9. Ma J, Mai HQ, Hong MH et al (2001) Results of a prospective randomized trial comparing neoadjuvant chemotherapy plus radiotherapy with radiotherapy alone in patients with locoregionally advanced nasopharyngeal carcinoma. J Clin Oncol 19:1350-35

10. Al-Sarraf M (2007) The role of concurrent chemo-radiotherapy in patients with head and neck cancers: a review. Gulf J Oncol 2:8-16

11. Al-Sarraf M, El-Hariry I (2008) The role of induction chemotherapy in the treatment of patients with locally advanced head and neck cancers: a review. Gulf J Oncol 4:8-18

12. Al-Sarraf M, Balaraman, S (2005) Head and Neck Cancer. In Giaccone G, Schilsky R, Sondel P (eds) Cancer chemotherapy and biological response modifiers. Annual 22, Chapter 18. Elsevier Science B.V. Amsterdam, pp 401-411

13. Al-Sarraf M, LeBlanc M, Giri PGS, Fu K, Cooper J, Vuong T, Forastiere A, Adams G, Sakr W, Schuller D, Ensley J (1996) Superiority of chemoradiotherapy (CT-RT) vs radiotherapy (RT) in patients (PTS) with locally advanced nasopharyngeal cancer (NPC). Preliminary results of Intergroup (0099) (SWOG 8892, RTOG 8817, ECOG 2388) randomized study. Plenary Session, Thirty-Second Annual Meeting, American Society of Clinical Oncology, May 18-21, Philadelphia, PA

14. Giri PGS, LeBlanc M, Al-Sarraf M, Fu KK, Cooper J, Vuong T, Forastiere A, Adams G, Sakr W, Schuller D, Ensley J (1996) Improved survival with chemotherapy and radiation therapy versus radiation therapy alone in advanced nasopharyngeal cancer. Preliminary results of an intergroup randomized trial. Int 0099, SWOG 8892, RTOG 8817, ECOG 2388. 38th Annual ASTRO Meeting, October 27-November 1, Los Angeles, CA 